\title{
The Use of High-Volume Endorectal Balloon is Effective to Reduce Doses of Rectum and Bladder in Prostate Cancer Patients Treated with Linac-Based Stereotactic Body Radiotherapy
}

\author{
(1) Mete GÜNDOĞ, (D) Hatice BAŞARAN, (D) Okan ORHAN, (1) Kadir YARAY, (1) Tarkan AKSÖZEN, \\ (1) Esin KIRAZ, (D) Celalettin EROĞLU
}

Department of Radiation Oncology, Erciyes University, Faculty of Medicine, Kayseri-Turkey

\begin{abstract}
OBJECTIVE
In this study, the effect of endorectal balloon (ERB) on risky organ doses was investigated in patients with low-medium risk prostate cancer treated with Stereotactic body radiotherapy (SBRT).

\section{METHODS}

In the 17 patients, CT simulation and treatment was performed with an empty rectum and no ERB was used (non-ERB group). ERB filled with 100-150 cc air into 15 patients was placed in the rectum and identified with the ERB group. SBRT was performed to be delivered with volumetric modulated arc, with 10 FFF photon beams, using a linear accelerator with a high definition MLC. Dosimetric parameters for the rectum, bladder and penilbulb were compared between the two groups as a retrospective. Independent samples t-tests were performed to examine the differences between the two groups.

\section{RESULTS}

The mean dose given to the rectum was lower in the ERB group than in the non-ERB group, and a statistically significant dose reduction of $149.4 \mathrm{cGy}$ per fraction was found in the ERB group $(\mathrm{p}<0.01)$. Similarly, the mean dose given to the bladder was lower in the ERB group than in the non-ERB group, and a statistically significant dose reduction of $178.8 \mathrm{cGy}$ per fraction was found in the ERB group $(\mathrm{p}<0.01)$. The mean dose of penilbulb was $373 \mathrm{cGy}$ in the ERB group, while the mean dose of penilbulb was 1314 $c G y$ in the non-ERB group. There was statistically significant differences between groups $(\mathrm{p}<0.01)$.
\end{abstract}

\section{CONCLUSION}

The use of high-volume ERB reduces rectum, bladder and penilbulb doses in prostate cancer patients treated with linac-based SBRT.

Keywords: Endorectal balloon; organ at risk; prostate cancer; SBRT.

Copyright $\odot$ 2020, Turkish Society for Radiation Oncology

\section{Introduction}

Among men, prostate cancer is the most common malignancy in developed countries.[1] The National
Comprehensive Cancer Network (NCCN) has defined a variety of management approaches, such as external beam radiotherapy (EBRT), brachytherapy, surgery, and active surveillance in low-risk or medi-

\section{Dr. Mete GÜNDOĞ}

Erciyes Üniversitesi Tıp Fakültesi,

Radyasyon Onkolojisi Anabilim Dalı,

Kayseri-Turkey

E-mail:mgundog@yahoo.com 
um-risk disease.[2] Oncological outcomes are similar in low-risk and intermediate-risk diseases, independent of treatment choice.[3] Besides, side-effects, such as sexual, gastrointestinal and genitourinary, may influence decision making of the treatment.[4] The evidence-based on prostate cancer shows that it has a low $\alpha / \beta$ ratio.[5] Hypo-fractional stereotactic body radiation therapy (SBRT), which improves accuracy in target localization and radiation dose distribution, has become increasingly used in recent years.[6-11] In SBRT for prostate cancer, it is crucial to reduce prostate movement due to the use of much higher doses and smaller target volumes per fraction, as well as to minimize the volume of rectum exposed to medium and high dose. There were studies (three non-inferiority studies and one superiority study) that comparing conventional and moderate hypofractionation for prostate cancer.[12-15] These studies pointed out that moderate hypofractionation was safe and results were comparable to conventional fractionation radiotherapy. Two prospective non-randomized trials comparing conventional fractionation and ultra-hypofractionation in a low-risk prostate cancer population have shown that high biochemical outcomes can be achieved with low toxicities.[16,17] Some studies have been published showing that restricting the movement of the prostate during treatment and removing the posterior part of the rectal wall from high dose areas may be possible using an ERB.[18-20] This practice can help reduce rectal toxicity by protecting the rectal wall from a medium-high radiation dose in patients undergoing post-prostatectomy or definitive radiotherapy.[21,22] However, the use of ERB is not compulsory in treatment protocols, and also the standardization of ERB has not yet been defined. The use of ERB has remained an option by clinicians. In this retrospective study, the effect of high volume ERB on the dose prescribed to the prostate and organ at risk doses were investigated in patients undergoing Linac-based SBRT therapy. The necessary permits for this study were obtained from the local ethics committee.

\section{Materials and Methods}

The data of thirty-two patients with low-intermediated risk prostate cancer, who were treated by SBRT in single-center, were evaluated retrospectively. All patients were simulated with a full bladder. Simulation CTs with a $2 \mathrm{~mm}$ thickness axial slice was acquired. The CT simulation and treatment of seventeen patients were administered without the use of ERB, and these pa- tients were identified as groups without ERB. For patients in the without-ERB group, to provide an empty rectum, they were given the use of laxative syrup and dietary advice for bowel preparation before simulation and before treatment. Fifteen patients whose simulations and treatments were performed using ERB were identified as with-ERB groups. In the with-ERB group, just before the CT simulation scan, an ERB (Q Fix, Indian) filled with $100-150 \mathrm{cc}$ of air was applied to the rectum. A multi-parametric MRI was performed for diagnostic and staging purposes for all patients. Then, the second MRI scans (with or without ERB) were taken using the immobilization apparatus suitable for the linac device and in the treatment position on a flat table. The simulation CTs were rigidly fused with T2-weighted sequences of this second MRI using the treatment planning system (Eclipse v.13.6, Varian Medical Systems, Palo Alto, USA). Clinical target volume (CTV) was defined as the entire prostate and the proximal portion of seminal vesicle for the low-risk group. The prostate plus $1 \mathrm{~cm}$ of the proximal seminal vesicles was defined as a CTV for intermediate-risk group. Instead of shaping the walls of the bladder and rectum, they were shaped as solid organs. The bladder was contoured from its base to the dome. The rectum was contoured from recto-sigmoid flexure to anus. Isotropic $3 \mathrm{~mm}$ was added to CTV to create The dose constraints were defined as follows; (I) Planning Target Volume (PTV) was prescribed to receive $36.25 \mathrm{cGy}$ in five fractions, (II) minimal dose coverage of the PTV of $98 \%$ (at least $95 \%$ of the PTV has to receive $100 \%$ of the prescribed dose), with a maximum dose of $<115 \%$ of the prescribed dose (39.8Gy) allowed. (III) The maximum rectal wall dose (Dmax rectal wall) should be less than $100 \%$ of the prescribed dose (36.25 Gy) and the highest dose defined to the $1 \mathrm{cc}$ rectum should not exceed $85 \%$ of the prescribed dose, (IV) the maximum bladder dose should not exceed 110\% (39.8 Gy) of the prescribed dose, and the highest dose to the $1 \mathrm{cc}$ bladder should be limited to $100 \%$ (36.25) of the prescribed dose. Before each treatment, verification of localization of the prostate was accomplished by a kilovoltage Cone-Beam Computerized Tomography (CBCT) scan and fusing it to the simulation CT. For the with-ERB group, ERB's position was evaluated before each treatment and after imaging with $\mathrm{CBCT}$, if radiological and clinically suitable, the installation was accepted and treatment was delivered. However, if ERB placement was not suitable, the set-up was not accepted, and it repeated. SBRT was applied by 10 FFF photon beams provided by a TrueBeam linear accelerator with high 
definition MLC (Varian medical systems, Palo Alto, USA) and with two full volumetric arcs. Dosimetric parameters were compared between the two groups for the organ at risk, including the mean dose, the maximum dose and the dose of 5\%, 20\%, 40\%, 50\%, $80 \%$, $90 \%$, and $100 \%$ of volume (Fig 1).

\section{Statistical Analysis}

Descriptive statistics were defined for each dosimetric parameter between the with-ERB group and the without-ERB group. Dosimetric data were converted to logarithmic ( $\log 10)$ to provide this necessary distribution so that the differences between groups could be calculated in independent sample t-tests. Mean and standard deviation values obtained from independent $\mathrm{t}$-tests were founded and the differences in the means value were shown as $\Delta$ for each parameter. Kruskal-Wallis test was performed to compare the averages of more than two groups. SPSS software was used for statistically analyses (version 21.0, SPSS Inc., Chicago, IL, USA). $\mathrm{p}<0.05$ was accepted as statistically significant.

\section{Results}

The median age of all patients was 68 (range 53-80). The median prostate volume was $44.7 \mathrm{cc}$ (range 20.7133.6) for all patients. In this study, 23 patients (71.9\%) had Gleason Score $<7$ and nine patients $(29.1 \%)$ had Gleason Score=7. The compliance of the patients to high-volume ERB was excellent. All 75 high-volume ERB applications were performed as planned. No patient gave up high-volume ERB administration. The median of the PTV-mean dose was found $37.76 \mathrm{~Gy}$ (range 36.25-38.16) for the with-ERB group, and the median of the PTV-mean dose was found 37.77 Gy (range 36.25-38.16) for the without-ERB group $(\mathrm{p}=0.44)$. The median prostate volume was found 45.2cc (range 27.9-133.6) in the with-ERB group and was found $43.1 \mathrm{cc}$ (range 20.7-94.7) in the withoutERB group $(p=0.47)$. The median of the PTV-max dose was found $40.27 \mathrm{~Gy}$ (range 38.18-41.74) for the with-ERB group, and the median of the PTV-max dose was found 39.04 Gy (range 36.89-41.26) for the without-ERB group. There was a statistically significant difference between the groups, according to PTV-max $(\mathrm{p}=0.01)$. The comparisons of the clinical features of the patients between the groups are shown in Table 1.

The mean dose to the rectum was lower in the withERB group than in the without-ERB group, and there was found statistically significant dose reduction 149.4 $c G y$ per fraction in the with-ERB group for mean rectal dose $(\mathrm{p}<0.01)$. As expected, the maximum dose administered to the rectum was higher in the with-ERB group than in the without-ERB group and an increase of $21.6 \mathrm{cGy}$ per fraction was statistically significant for the maximum rectal dose in the with-ERB group $(\mathrm{p}=0.01)$. The average doses of rectum $\mathrm{V} \% 5, \mathrm{~V} \% 20$, V\%40, V50\%, V80\%, V90\%, and V100\%; in the with-

Table 1 Comparison of the clinical characteristics of the patients between groups

\begin{tabular}{|c|c|c|c|c|c|}
\hline & \multicolumn{2}{|c|}{$\begin{array}{l}\text { SBRT with ERB group } \\
\qquad(n=15)\end{array}$} & \multicolumn{2}{|c|}{$\begin{array}{l}\text { SBRT without ERB group } \\
\qquad(n=17)\end{array}$} & \multirow[b]{2}{*}{$\mathbf{p}$} \\
\hline & Median & Range & Median & Range & \\
\hline Prostate (cc) & 45.2 & $27.9-133.6$ & 43.1 & $20.7-94.7$ & $0.47^{*}$ \\
\hline $\mathrm{Cl}$ & 1.04 & $0.9-1.3$ & 0.99 & $0.7-1.19$ & $0.03^{*}$ \\
\hline PTV coverage & 95 & $82.4-99.0$ & 92 & $66-98$ & $0.11^{*}$ \\
\hline PTV mean dose (cGy) & 3776 & $3625-3816$ & 3737 & $3625-3816$ & $0.44^{*}$ \\
\hline PTV max dose (cGy) & 4027 & $3818-4174$ & 3904 & $3689-4126$ & $0.01 *$ \\
\hline Pre-SBRT PSA & 8 & $5-27.8$ & 10.1 & $0.3-38.17$ & $0.85^{*}$ \\
\hline \multicolumn{6}{|l|}{ Gleason score } \\
\hline Gleason $<7$ & 9 & $60(\%)$ & 14 & $82.4(\%)$ & $0.13^{* *}$ \\
\hline Gleason=7 & 6 & $40(\%)$ & 3 & $17.6(\%)$ & \\
\hline \multicolumn{6}{|l|}{ Risk group } \\
\hline Low-risk & 9 & $60(\%)$ & 11 & $64.7(\%)$ & $1.00^{* *}$ \\
\hline Intermediate-risk & 6 & $40(\%)$ & 6 & $35.3(\%)$ & \\
\hline
\end{tabular}

Cl: Conformity index; PTV: Planning target volume; SBRT: Stereotactic body radiosurgery; ERB: Endorectal balloon *Mann-Whitney U test value; **Chi-Square test value 
ERB Group were 30.2 Gy, 16.2 Gy, 511.3 Gy, 8.9 Gy, 2.4 Gy, 1.35 Gy, and 0.4 Gy compared with the withoutERB group were 34.2 Gy, 25.1 Gy, 17.2 Gy, 14.1 Gy, 7.1 Gy, 5.1 Gy and 2.0 Gy respectively The dose administered to the volumes of Rectum V\%20, V\%40, V\%50, $\mathrm{V} \% 80, \mathrm{~V} \% 90$ and V\%100 were statistically lower in the with-ERB group compared to the without-ERB group $(\mathrm{p}<0.01, \mathrm{p}<0.01, \mathrm{p}<0.01, \mathrm{p}<0.01, \mathrm{p}<0.01$ and $\mathrm{p}<0.01$ respectively). The mean dose to given to the rectum was lower in the with-ERB group than in the withoutERB group and there was found statistically significant dose reduction $149.4 \mathrm{cGy}$ per fraction in the with-ERB group $(\mathrm{p}<0.01)$. However, there was no difference between the groups in terms of V\%5 ( $\mathrm{p}=0.57)$. On the other hand, a pattern of dose reduction was observed between the with-ERB Group and the without-ERB Group concerning the dose administered to the bladder. The average doses of bladder V\%5, V\%20, V\%40, V50\%, V80\%, V90\%, and V100\%; in the with-ERB Group were 25.3 Gy, 11.3 Gy, 5.5 Gy, 4.2Gy, 1.5 Gy, 0.9 Gy, and 0.4 Gy compared with the without-ERB group were 34.2 Gy, 25.1 Gy, 17.2 Gy, 14.1 Gy, 7.1 Gy, 5.1 Gy and 2.0 Gy, respectively. The doses of the bladder in the with-ERB group were significantly lower than in the without-ERB group $(p<0.01)$. Especially, the mean dose to administer to the bladder was lower in the with-ERB group than in the without-ERB group and there was found statistically significant dose reduction $178.8 \mathrm{cGy}$ per fraction in the with-ERB group $(\mathrm{p}<0.01)$. However, there was no statistically significant difference between the groups in terms of the maximum dose of the bladder $(p=0.43)$. The comparisons of the dosimetric differences between the groups are shown in Table 2. The DVH profile comparison between the with-ERB group and the without-ERB group for the volume of rectum and bladder in the low to-high dose region are shown in Figure 2.

Concerning mean penile bulb dose, statistically significantly a difference was found between groups $(p<0.01)$. The mean dose of the penile bulb was 373 cGy in the with-ERB group, while the mean dose of the penile bulb was $1314 \mathrm{cGy}$ in the without-ERB

Table 2 The comparisons of the dosimetric differences between the groups

\begin{tabular}{|c|c|c|c|c|c|c|c|}
\hline & \multicolumn{2}{|c|}{$\begin{array}{c}\text { With-ERB group }(n=15) \\
\text { Dose }(c G y)\end{array}$} & \multicolumn{2}{|c|}{$\begin{array}{c}\text { Without-ERB group }(n=17) \\
\text { Dose (cGy) }\end{array}$} & \multirow[b]{2}{*}{$\Delta$ (cGy) } & \multirow[b]{2}{*}{$\Delta$ (cGy)/fr } & \multirow[b]{2}{*}{$\mathbf{p}$} \\
\hline & Mean \pm SD & $(\log 10)$ & Mean \pm SD & $(\log 10)$ & & & \\
\hline \multicolumn{8}{|l|}{ Rectum } \\
\hline Mean dose & $10.68 \pm 2.2$ & $(3.01 \pm 0.09)$ & $18.16 \pm 3.1$ & $(3.25 \pm 0.07)$ & 747 & 149.4 & $<0.01$ \\
\hline Max dose & $38.1 \pm 1.14$ & $(3.58 \pm 0.01)$ & $37.0 \pm 1.14$ & $(3.56 \pm 0.01)$ & 108 & 21.6 & 0.01 \\
\hline $\mathrm{V}_{\% 5}$ & $30.2 \pm 4.2$ & $(3.47 \pm 0.06)$ & $30.8 \pm 2.8$ & $(3.48 \pm 0.04)$ & 57 & 11.4 & 0.57 \\
\hline $\mathrm{V}_{\% 20}$ & $16.2 \pm 3.8$ & $(3.19 \pm 0.11)$ & $24.8 \pm 2.6$ & $(3.39 \pm 0.04)$ & 860 & 172 & $<0.01$ \\
\hline $\mathrm{V}_{\% 40}$ & $11.3 \pm 3.5$ & $(3.03 \pm 0.15)$ & $20.6 \pm 2.9$ & $(3.31 \pm 0.06)$ & 931 & 186.2 & $<0.01$ \\
\hline $\mathrm{V}_{\% 50}$ & $8.91 \pm 4.1$ & $(2.89 \pm 0.23)$ & $18.8 \pm 3.5$ & $(3.26 \pm 0.09)$ & 991 & 198.2 & $<0.01$ \\
\hline $\mathrm{V}_{\% 80}$ & $2.4 \pm 1.2$ & $(2.32 \pm 0.23)$ & $10.9 \pm 5.9$ & $(2.93 \pm 0.37)$ & 851 & 170.2 & $<0.01$ \\
\hline $\mathrm{V}_{\% 90}$ & $1.35 \pm 0.6$ & $(2.08 \pm 0.21)$ & $6.48 \pm 4.9$ & $(2.66 \pm 0.39)$ & 512 & 102.4 & $<0.01$ \\
\hline$V_{\% 100}$ & $0.4 \pm 0.08$ & $(1.59 \pm 0.08)$ & $2.07 \pm 1.9$ & $(2.19 \pm 0.31)$ & 166 & 33.2 & $<0.01$ \\
\hline \multicolumn{8}{|l|}{ Bladder } \\
\hline Mean dose & $7.16 \pm 4.0$ & $(2.79 \pm 0.24)$ & $16.1 \pm 5.0$ & $(3.18 \pm 0.14)$ & 894 & 178.8 & $<0.01$ \\
\hline Max dose & $38.56 \pm 1.9$ & $(3.58 \pm 0.02)$ & $38.1 \pm 0.9$ & $(3.58 \pm 0.01)$ & 46 & 9.2 & 0.43 \\
\hline $\mathrm{V}_{\% 5}$ & $25.3 \pm 6.2$ & $(3.39 \pm 0.11)$ & $34.2 \pm 3.2$ & $(3.53 \pm 0.04)$ & 896 & 179.2 & $<0.01$ \\
\hline $\mathrm{V}_{\% 20}$ & $11.3 \pm 7.3$ & $(2.93 \pm 0.37)$ & $25.1 \pm 6.2$ & $(3.38 \pm 0.11)$ & 1378 & 275.6 & $<0.01$ \\
\hline$V_{0 \% 40}$ & $5.5 \pm 5.5$ & $(2.51 \pm 0.46)$ & $17.2 \pm 6.2$ & $(3.20 \pm 0.17)$ & 1171 & 234.2 & $<0.01$ \\
\hline $\mathrm{V}_{\% 50}$ & $4.2 \pm 4.8$ & $(2.36 \pm 0.48)$ & $14.1 \pm 6.4$ & $(3.08 \pm 0.26)$ & 983 & 196.6 & $<0.01$ \\
\hline $\mathrm{V}_{\% 80}$ & $1.5 \pm 1.6$ & $(1.99 \pm 0.39)$ & $7.1 \pm 5.5$ & $(2.69 \pm 0.41)$ & 562 & 112.4 & $<0.01$ \\
\hline$V_{\% 90}$ & $0.9 \pm 0.7$ & $(1.85 \pm 0.34)$ & $5.1 \pm 4.7$ & $(2.52 \pm 0.42)$ & 415 & 83 & $<0.01$ \\
\hline $\mathrm{V}_{\% 100}$ & $0.4 \pm 0.3$ & $(1.58 \pm 0.28)$ & $2.0 \pm 2.8$ & $(2.12 \pm 0.37)$ & 161 & 32.2 & $<0.01$ \\
\hline \multicolumn{8}{|l|}{ Penilbulb } \\
\hline Mean dose & $3.7 \pm 1.9$ & $(2.51 \pm 0.24)$ & $6.5 \pm 3.7$ & $(2.75 \pm 0.24)$ & 284 & 56.8 & $<0.01$ \\
\hline Max dose & $13.1 \pm 9.1$ & $(3.00 \pm 0.33)$ & $17.8 \pm 8.8$ & $(3.19 \pm 0.24)$ & 468 & 93.6 & 0.07 \\
\hline
\end{tabular}

ERB: Endorectal balloon, $\triangle$ : Mean difference between groups, p: Independent-samples T-Test value, SD: Standard deviation 

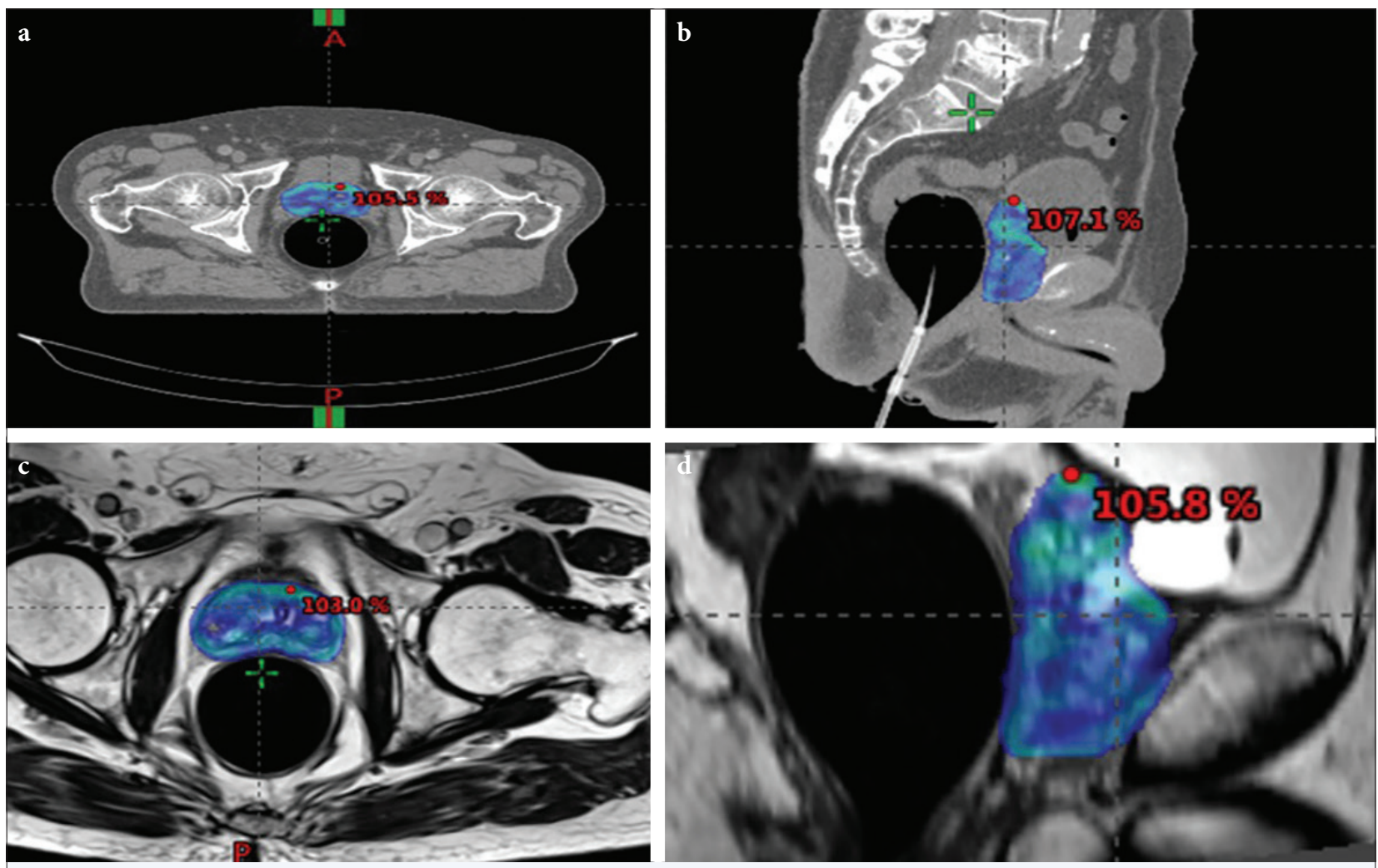

Fig. 1. The delivered dose distribution to the target volume. (a) The axial image on the CT slice. (b) The sagittal image on the CT slice. (c) The axial image on MRI slice. (d) The sagittal image on MRI slice.
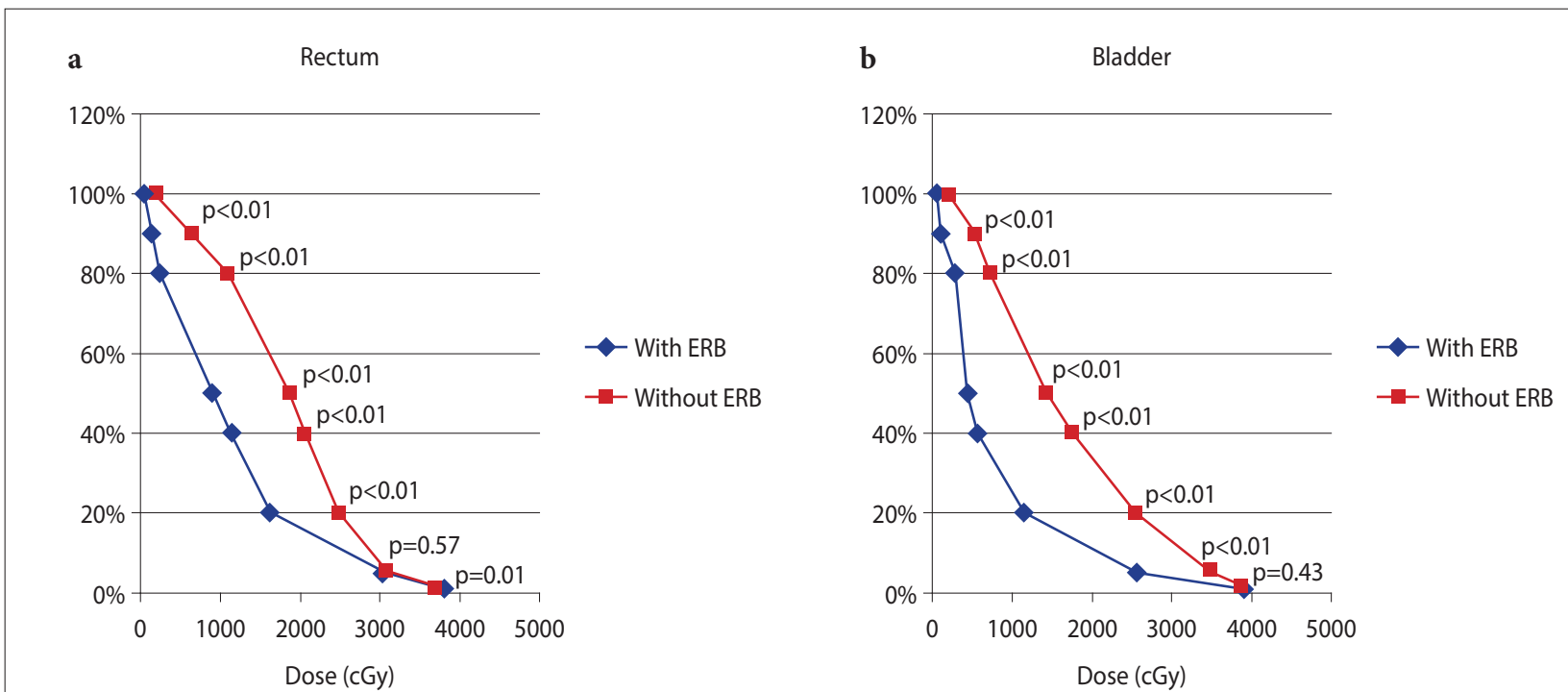

Fig. 2. The comparison of the organ at risk doses between the groups that were treated with and without the endorectal balloon. (a) For rectum. (b) For Bladder.

group. Concerning mean rectum doses of patients between the low-risk group and the intermediate-risk group, the difference between the groups was not sta- tistically significant $(\mathrm{p}=0.60)$. The mean rectum dose differences between subgroups are summarized in Table 3. 
Table 3 Multiple comparison of the subgroups regarding rectum doses

\begin{tabular}{|c|c|c|c|c|c|c|}
\hline & & & Test statistic & SE & $\Delta$ & $\mathbf{p}^{*}$ \\
\hline Low risk and ERB & $\mathrm{v}$ & Intermediate risk and ERB & 5.222 & 4.9 & 260.1 & 0.29 \\
\hline Intermediate risk and ERB & $\mathrm{v}$ & Low risk and No-ERB & 17.677 & 4.6 & 883.8 & $<0.01$ \\
\hline Intermediate risk and ERB & $\mathrm{v}$ & Intermediate risk and No-ERB & 19.967 & 5.6 & 950.7 & $<0.01$ \\
\hline Low risk and ERB & v & Low risk and No-ERB & 12.444 & 4.1 & 623.6 & 0.01 \\
\hline Low risk and ERB & v & Intermediate risk and No-ERB & 14.444 & 5.2 & 690.6 & 0.03 \\
\hline Low risk and No-ERB & v & Intermediate risk and No-ERB & -2.000 & 4.9 & 66.9 & 0.68 \\
\hline
\end{tabular}

ERB: Endorectal balloon; SE: Standard error; $\Delta$ : Mean difference between groups (cGy): * $\mathrm{p}$ : Kruskal-Wallis test value

\section{Discussion}

In addition to improved treatment delivery and improvements in image-guided radiotherapy, daily inserted ERB is used to immobilize the prostate and to reduce CTV-PTV margins.[22] ERB administration may have a protective effect, particularly by pushing the posterior rectum away from the high-dose areas. [23] For prostate cancer, the use of ERB to reduce the dose to the rectal wall has been reported many times with 3D-CRT and IMRT experience. Patel et al. first reported that using ERB in 3D-CRT, which resulted in dose reduction comparable to IMRT for rectal protection.[24] After this study, the idea has come to minds that more protection can be achieved using a rectal balloon in IMRT. Subsequently, studies were presented that using ERB resulted in a reduction of the rectal dose. Smeenk et al. showed that the use of $80 \mathrm{cc}$ ERB, in patients with treated both 3D CRT and IMRT, resulted in a decrease in rectal doses.[25] Additionally, Van Lin et al. detected a decrease in rectal doses in patients treated with 3Dcrt in their study using $40 \mathrm{cc}, 80 \mathrm{cc}$, and $100 \mathrm{cc}$ ERB, while they did not show a reduction in rectal doses in patients treated with IMRT.[26] The results of the Phase-3 HYPRO study investigating the efficacy and toxicity of moderate fractionated radiotherapy showed that reduction of anorectal dose and gastrointestinal toxicity were possible by MRI delineation of the prostate and ERB insertion.[27] The studies in the literature pointed out those ERB preferences with high levels were superior concerning spearing normal tissues.[28] However, the use of ERB in clinical practice requires additional time, especially in long-term therapy plans and in intensive clinics. Using ERB may extend the time required for treatment setup, and it is required re-correction due to incorrect inflation. Thus, the combination of ERB and stereotactic body surgery may be more preferable. There are a few studies in the literature which are about this togetherness. A study proved that using ERB results in reductions in prostate deformations in their trial, which was delivered a total of 50 Gy in five fractions with Linac-based SBRT. [29] In the same trial the researchers also observed that there was no statistically significant dose reduction for bladder and anterior rectum walls.[29] However, that researchers used low-volume ERB $(60 \mathrm{cc})$ in this trial may not have achieved the desired dose reduction. In robotic-SBRT experience, which was performed by Xiang HF et al., and was delivered 36.25 Gy in five fractions, observed that there was significant rectal wall dose reduction using ERB.[30] In their studies, they preferred the volume of 60-100 cc ERB, and the combination of higher ERB volume helped reduce the doses in rectum walls. Jaccard et al. represented that mean dose decreasing in internal pudendal arteries was available by ERB (100 cc) insertion. However, there was no decrease of doses in volumes of the rectal wall with V\%100, V\%90, V\%80 and also there was increasing in the dose of the bladder wall with V\%100. [31] In this current study, we demonstrated that using high volume ERB (100-150 cc) in per-fraction in treatment provides dose reduction for rectal wall volumes with V\%100, V\%90, V\%80, V\%50, V\%40 and V\%20. As expected, the maximum rectal wall dose was increased in patients treated with high volume ERB. In contrast to some studies in the literature, we observed that reductions of doses were achieved using ERB in the bladder wall. We preferred to use high volume ERB during simulation and treatment $(100-150 \mathrm{cc})$. However, to our knowledge, none of the studies in the literature have specified the use of high-volume ERB as in our study. In contrast to the trial of Bones et al., in our linac-based study, significant dose reductions in the rectum were found. The difference between ERB volumes may have led to a different result between these two studies. However, it is not clear which ERB volume is standard concerning tolerability and effectiveness in the literature. In patients with localized prostate cancer, 
expected overall survival is long. Therefore, evaluation and management of toxicities, especially concerning genitourinary and anorectal, is significant for the quality of life. Application of high volume ERB may assist us in protecting organs at risk like the rectum, bladder, and penile bulb. Hypothetically, ERB with high volumes may establish hypoxia in the intramural layer of the rectum using increasing pressure. Therefore, releasing free oxygen radical levels may decrease. In addition to providing dose reduction in organ-at-risk, ERB may increase the radioprotective effect by increasing hypoxia with increased pressure and reducing free-oxygen radicals for rectum tissue. There are some limitations, such as limited patient numbers, the uncertainty of intra-fraction motion, and no evaluation of toxicity because of low patient numbers in this current study.

\section{Conclusion}

Consequently, we observed a significant dose of reduction in prostate cancer patients treated with Linac-based SBRT for rectum, bladder, and penile bulb using ERB. Further, both clinical and pre-clinical studies are needed to evaluate the dosimetric and radiobiological advantages of ERB.

Peer-review: Externally peer-reviewed.

Conflict of Interest: The authors declared that they have no conflict interests.

Ethics Committee Approval: This study has been approved by the local ethics committee (2019/833).

Financial Support: This research received no specific grant from any funding agency in the public, commercial, or notfor-profit sectors.

Authorship contributions: Concept - M.G.; Design M.G.; Supervision - H.B., E.K.; Funding - None; Materials - K.Y., T.A.; Data collection and/or processing - H.B., E.K., O.O..; Data analysis and/or interpretation - M.G; Literature search - M.G., H.B., C.E., E.K., O.O.; Writing - M.G.; Critical review - O.O., C.E.

\section{References}

1. Brand DH, Tree AC, Ostler P, van der Voet H, Loblaw $\mathrm{A}, \mathrm{Chu} \mathrm{W}$, et al. Intensity-modulated fractionated radiotherapy versus stereotactic body radiotherapy for prostate cancer (PACE-B): acute toxicity findings from an international, randomised, open-label, phase 3, non-inferiority trial. Lancet Oncol 2019;20(11):1531-43.

2. National Comprehensive Cancer Network.NCCN clinical practice guidelines in oncology: prostate cancer. Available at: https://www.nccn.org/professionals/ physician_gls/pdf/prostate.pdf. Accessed Aug 1, 2018.

3. Hamdy FC, Donovan JL, LaneJA, etal. 10-Year Outcomes after Monitoring, Surgery, or Radiotherapy for Localized Prostate Cancer. N Engl J Med 2016;375(15):1415-24.

4. Donovan JL, Hamdy FC, Lane JA, Mason M, Metcalfe C, Walsh E, et al. Patient-Reported Outcomes after Monitoring, Surgery, or Radiotherapy for Prostate Cancer. N Engl J Med 2016;375(15):1425-37.

5. Miralbell R, Roberts SA, Zubizarreta E, Hendry JH. Dose-fractionation sensitivity of prostate cancer deduced from radiotherapy outcomes of 5,969 patients in seven international institutional datasets: $\alpha / \beta=1.4(0.9-2.2)$ Gy. Int J Radiat Oncol Biol Phys 2012;82(1):17-24.

6. De Los Santos J, Popple R, Agazaryan N, Bayouth JE, Bissonnette JP, Bucci MK, et al. Image guided radiation therapy (IGRT) technologies for radiation therapy localization and delivery. Int $J$ Radiat Oncol Biol Phys 2013;87(1):33-45.

7. Madsen BL, Hsi RA, Pham HT, Fowler JF, Esagui L, Corman J. Stereotactic hypofractionated accurate radiotherapy of the prostate (SHARP), 33.5 Gy in five fractions for localized disease: first clinical trial results. Int J Radiat Oncol Biol Phys 2007;67(4):1099-105.

8. King CR, Brooks JD, Gill H, Pawlicki T, Cotrutz C, Presti JC Jr. Stereotactic body radiotherapy for localized prostate cancer: interim results of a prospective phase II clinical trial. Int J Radiat Oncol Biol Phys 2009;73(4):1043-8.

9. Freeman DE, King CR. Stereotactic body radiotherapy for low-risk prostate cancer: five-year outcomes. Radiat Oncol 2011;6:3.

10. McBride SM, Wong DS, Dombrowski JJ, Harkins B, Tapella P, Hanscom HN, et al. Hypofractionated stereotactic body radiotherapy in low-risk prostate adenocarcinoma: preliminary results of a multi-institutional phase 1 feasibility trial. Cancer 2012;118(15):3681-90.

11. King CR, Brooks JD, Gill H, Presti JC Jr. Long-term outcomes from a prospective trial of stereotactic body radiotherapy for low-risk prostate cancer. Int J Radiat Oncol Biol Phys 2012;82(2):877-82.

12. Dearnaley D, Syndikus I, Mossop H, Khoo V, Birtle A, Bloomfield D, et al. Conventional versus hypofractionated high-dose intensity-modulated radiotherapy for prostate cancer: 5-year outcomes of the randomised, non-inferiority, phase $3 \mathrm{CHHiP}$ trial. Lancet Oncol 2016;(8):1047-60.

13. Lee WR, Dignam JJ, Amin MB, Bruner DW, Low D, Swanson GP, et al. Randomized Phase III Noninferiority Study Comparing Two Radiotherapy Fractionation Schedules in Patients With Low-Risk Prostate Cancer. J Clin Oncol 2016;34(20):2325-32. 
14. Catton CN, Lukka H, Gu CS, Martin JM, Supiot S, Chung PWM, et al. Randomized Trial of a Hypofractionated Radiation Regimen for the Treatment of Localized Prostate Cancer. J Clin Oncol 2017;35(17):1884-90.

15. Incrocci L, Wortel RC, Alemayehu WG, Aluwini S, Schimmel E, Krol S, et al. Hypofractionated versus conventionally fractionated radiotherapy for patients with localised prostate cancer (HYPRO): final efficacy results from a randomised, multicentre, open-label, phase 3 trial. Lancet Oncol 2016;17(8):1061-9.

16. Musunuru HB, Quon H, Davidson M, Cheung P, Zhang L, D'Alimonte L, et al. Dose-escalation of five-fraction SABR in prostate cancer: Toxicity comparison of two prospective trials. Radiother Oncol 2016;118(1):112-7.

17. Loblaw A, Pickles T, Crook J, Martin AG, Vigneault E, Souhami L, et al. Stereotactic Ablative Radiotherapy Versus Low Dose Rate Brachytherapy or External Beam Radiotherapy: Propensity Score Matched Analyses of Canadian Data. Clin Oncol (R Coll Radiol) 2017;29(3):161-70.

18. Both S, Wang KK, Plastaras JP, Deville C, Bar Ad V, Tochner Z, et al. Real-time study of prostate intrafraction motion during external beam radiotherapy with daily endorectal balloon. Int J Radiat Oncol Biol Phys 2011;81(5):1302-9.

19. Smeenk RJ, Louwe RJ, Langen KM, Shah AP, Kupelian $\mathrm{PA}$, van Lin EN, et al. An endorectal balloon reduces intrafraction prostate motion during radiotherapy. Int J Radiat Oncol Biol Phys 2012;83(2):661-9.

20. Wang KK, Vapiwala N, Deville C, Plastaras JP, Scheuermann R, Lin H, et al. A study to quantify the effectiveness of daily endorectal balloon for prostate intrafraction motion management. Int J Radiat Oncol Biol Phys 2012;83(3):1055-63.

21. Smeenk RJ, van Lin EN, van Kollenburg P, McColl GM, Kunze-Busch M, Kaanders JH. Endorectal balloon reduces anorectal doses in post-prostatectomy intensity-modulated radiotherapy. Radiother Oncol 2011;101(3):465-70.

22. Smeenk RJ, Teh BS, Butler EB, van Lin EN, Kaanders $\mathrm{JH}$. Is there a role for endorectal balloons in prostate radiotherapy? A systematic review. Radiother Oncol 2010;95(3):277-82.

23. Wachter S, Gerstner N, Dorner D, Goldner G, Colotto
A, Wambersie A, et al. The influence of a rectal balloon tube as internal immobilization device on variations of volumes and dose-volume histograms during treatment course of conformal radiotherapy for prostate cancer. Int J Radiat Oncol Biol Phys 2002;52(1):91-100.

24. Patel RR, Orton N, Tomé WA, Chappell R, Ritter MA. Rectal dose sparing with a balloon catheter and ultrasound localization in conformal radiation therapy for prostate cancer. Radiother Oncol 2003;67(3):285-94.

25. Smeenk RJ, van Lin EN, van Kollenburg P, Kunze-Busch M, Kaanders JH. Anal wall sparing effect of an endorectal balloon in 3D conformal and intensity-modulated prostate radiotherapy. Radiother Oncol 2009;93(1):131-6.

26. van Lin EN, Hoffmann AL, van Kollenburg P, Leer JW, Visser AG. Rectal wall sparing effect of three different endorectal balloons in 3D conformal and IMRT prostate radiotherapy. Int J Radiat Oncol Biol Phys 2005;63(2):565-76.

27. Wortel RC, Heemsbergen WD, Smeenk RJ, Witte MG, Krol SDG, Pos FJ, et al. Local Protocol Variations for Image Guided Radiation Therapy in the Multicenter Dutch Hypofractionation (HYPRO) Trial: Impact of Rectal Balloon and MRI Delineation on Anorectal Dose and Gastrointestinal Toxicity Levels. Int J Radiat Oncol Biol Phys 2017;99(5):1243-52.

28. Vlachaki MT, Teslow TN, Amosson C, Uy NW, Ahmad S. IMRT versus conventional 3DCRT on prostate and normal tissue dosimetry using an endorectal balloon for prostate immobilization. Med Dosim 2005;30(2):69-75.

29. Jones BL, Gan G, Diot Q, Kavanagh B, Timmerman RD, Miften M. Dosimetric and deformation effects of image-guided interventions during stereotactic body radiation therapy of the prostate using an endorectal balloon. Med Phys 2012;39(6):3080-8.

30. Xiang HF, Lu HM, Efstathiou JA, Zietman AL, De Armas R, Harris K, et al. Dosimetric impacts of endorectal balloon in CyberKnife stereotactic body radiation therapy (SBRT) for early-stage prostate cancer. J Appl Clin Med Phys 2017;18(3):37-43.

31. Jaccard M, Lamanna G, Dubouloz A, Rouzaud M, Miralbell R, Zilli T. Dose optimization and endorectal balloon for internal pudendal arteries sparing in prostate SBRT. Phys Med 2019;61:28-32. 